\title{
Estudo da economia de escala do setor de telecomunicações móveis do Brasil pós-privatizações ${ }^{1}$
}

\section{Study of the scale economy in the brazilian mobile telecommunication sector after privatizations}

\author{
Maria Ivanice Vendruscolo \\ Professora Mestre do Departamento de Ciências Contábeis da Faculdade de Administração, Contabilidade e Economia \\ da Pontifícia Universidade Católica do Rio Grande do Sul • E-mail: m.ivanice@terra.com.br \\ Tiago Wickstrom Alves \\ Professor Doutor dos Programas de Pós-Graduação em Ciências Contábeis e Economia das Faculdades de Ciências Contábeis e Economia \\ da Universidade Vale Rio dos Sinos • E-mail: twa@unisinos.br
}

Recebido em 29.06.2007 • Aceito em 25.05.2008 • $2^{a}$ versão aceita em 04.08.2008

\section{RESUMO}

O setor de telecomunicações passou por significativas transformações estruturais, inovações tecnológicas e mudanças nos processos regulatórios nas últimas décadas, em nível mundial. A telefonia móvel brasileira seguiu a mesma tendência, desenvolvendo uma complexa estrutura oligopolista após a quebra do monopólio estatal. Inicialmente, com a abertura do mercado, foi adotada uma estrutura na qual a competição se dava entre operadoras de Banda A e B. Num segundo momento, com o cumprimento das metas de universalização, previstas pelo novo modelo de telecomunicações brasileiro, a concorrência entre as operadoras foi intensificada, com a entrada das operadoras de Banda D e E. Dadas as mudanças ocorridas na estrutura de mercado do setor de telefonia brasileiro, no período pós-privatizações, este estudo teve por objetivo verificar se as operadoras de telefonia móvel brasileiras estavam usufruindo economias de escala por meio da análise da função de custo polinomial e das curvas de custos. A pesquisa foi de natureza aplicada do tipo documental, utilizando o método estatístico de mínimos quadrados com um modelo para dados em painel. Os resultados estimados da função de custos para o setor revelaram que a produção com base no número de clientes apresentou retornos crescentes e decrescentes de produção, evidenciando a existência de economias de escala no setor. Entretanto, o setor encontrava-se com um número excessivo de empresas, dada a demanda do mercado para que as empresas de telefonia móvel brasileiras pudessem usufruir os benefícios dos ganhos de escala.

Palavras-chave: Economia de escala. Função de custos. Telecomunicações.

\section{ABSTRACT}

In recent decades, the telecommunication sector has gone through significant structural transformations, technological innovations and changes in regulatory processes around the world. In Brazil, after the state monopoly had been broken, mobile telephony followed the same trend, developing a complex oligopolistic structure. Initially, when the market opened, a structure was in which competition took place between $A$ and $B$ Band operators. In a second phase, when universalization targets were achieved, foreseen in the new Brazilian telecommunications model, competition between the operators was intensified, with the entrance of $D$ and $E$ Band operators. Given the changes in the market structure of the Brazilian telephony sector, in

10 artigo apresentado no $1^{\circ}$ Congresso ANPCONT, em Gramado-RS, 2007. 
the period after privatizations, this study aimed to verify if the Brazilian mobile telephony operators were enjoying economies of scale, through the analysis of the polynomial cost function and of cost curves. An applied documentary research was carried out, using the statistical method of least squares with a model for panel data. The estimated results of the cost function for the sector revealed that production based on the number of clients presented growing returns and decreasing production, evidencing the existence of economies of scale in the sector. However, the sector contained an excessive number of companies, given the market's demand for Brazilian mobile telephone companies to enjoy the benefits of scale gains.

Keywords: Economies of scale. Costs function. Telecommunications.

\section{INTRODUÇÃO}

O setor de telecomunicações em nível mundial passou por significativas transformações estruturais nas últimas décadas, como, por exemplo, mudanças tecnológicas, alterações nos processos regulatórios de mercado e nas relações comerciais da cadeia produtiva (NEVES, 2002). Modificações essas que, segundo Pessini (2005), foram decorrentes do desenvolvimento tecnológico que reduziu fortemente as barreiras de entradas mediante o barateamento dos novos meios de produção, permitindo a abertura do mercado à competição internacional.

A abertura do mercado de telecomunicações e a internacionalização do mercado de capitais aumentaram a atratividade para o capital estrangeiro. As grandes operadoras privadas americanas e européias intensificaram a concorrência mundial com a abertura do mercado brasileiro e realizaram fortes investimentos no Brasil. Em 1999, 79\% dos investimentos externos aplicados no Brasil foram realizados no segmento serviços, principalmente no setor de telecomunicações (RODRIGUES, 2000).

Novas empresas iniciaram operações de telefonia móvel no Brasil, aumentando a concorrência e um número maior de clientes passou a ser atendido pelos serviços ofertados. Esse crescimento decorreu principalmente de forte pressão competitiva entre as operadoras, que forçou a queda da barreira de entrada e do preço dos serviços.

Com isso, a telefonia brasileira ganhou destaque com os processos de privatizações e globalização da economia, promovendo avanços tecnológicos que possibilitaram a infra-estrutura necessária à obtenção de vantagem competitiva.

Uma das estratégias utilizadas para ganhar competitividade foi a formação de parcerias, principalmente motivadas pelos ganhos de escala. O aumento no número de empresas fez com que elas dirigissem seus esforços na redução de custos e ganhos de escala com a formação de joint venture, de forma a proporcionar às operadoras uma posição sustentável no mercado. Assim, a gestão dos custos operacionais passou a representar uma questão vital para as operadoras de telefonia no período pós-privatizações do setor.

Logo, devido as mudanças no mercado de telefonia brasileiro e com as transformações econômicas e tecnológicas que ocorreram nesse setor, intensificando a concorrência, questiona-se: o número de empresas de telefonia móvel no Brasil é adequado à demanda do mercado no que se refere a ganhos de escala? Ou, em outras palavras, essas empresas operam no ponto de mínimo da função de custos?

Tendo em vista essas questões é que se propõe este trabalho, cujo objetivo é o de verificar em que região da função de custo total do setor as empresas estão operando, com o intuito de analisar os ganhos de escala do setor por meio da análise das curvas de custo do setor. 


\section{REFERENCIAL TEÓRICO}

\subsection{Curvas de Custos}

As curvas de custos tratam da relação entre os custos e o nível de produção, evidenciando a variação do custo total com o aumento das quantidades produzidas. A curva de custo total de curto prazo mostra as combinações de custo total em determinada quantidade produzida, dada a tecnologia empregada na produção. Por meio da análise da curva de custo total de curto prazo, é possível escolher a combinação ótima de produção, ou seja, a que minimiza os custos.

Besanko e Braeutigam (2004, p. 219) esclarecem que a curva de custo de curto prazo "nos mostra o custo total mínimo de produção de $Q$ unidades de produto, quando a quantidade utilizada de pelo menos 1 insumo é constante" e que é igual à soma da curva de custo variável total e à curva de custo fixo total, ou seja:

$C T_{C P}(Q)=C V T(Q)+C F T$

em que:

$C T_{C P}=$ custo total de curto prazo;

$C V T=$ custo variável total;
CFT = custo fixo total;

$Q=$ quantidade.

Na Figura $1 \boldsymbol{\nabla}$, representam-se a curva de custo total, o custo total variável e o custo total fixo de curto prazo.

Explicando-se cada curva da Figura 1, supondo que o único insumo fixo no curto prazo seja o estoque de capital “ $k$ ”, ou seja, a quantidade de capital utilizada, tem-se:

- custos fixos totais (CFT): igual ao custo dos serviços de capital fixos " $k$ " sendo " $r$ ", o custo de capital e " $d$ " o custo de reposição do capital, então, $(r+d) K$ é o custo total do capital e será fixo; sua representação é uma linha paralela ao eixo das quantidades;

- custo variável total $C V T(Q)$ : o custo variável aumenta com o nível de quantidade produzida, assim a curva é inclinada para cima; porém não é linear, pois haverá uma região inicial em que os custos crescem a taxas decrescentes devido aos ganhos de escala e depois passam a crescer a taxas crescentes devido à absorção do insumo fixo, no caso o capital;

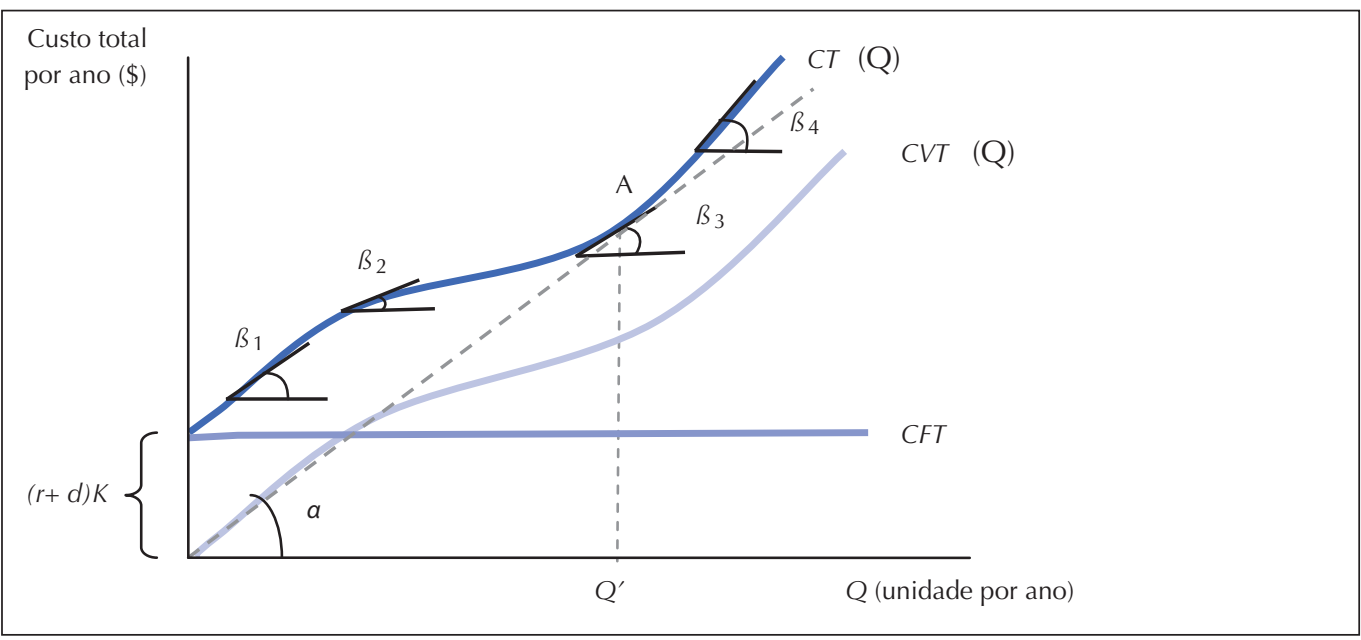

Fonte: Adaptado de Besanko e Braeutigam (2004, p. 220)

Figura 1 Curva de custos total de curto prazo 
- custo total é simplesmente a soma dos custos fixos e variáveis; assim, a distância vertical do custo total é igual à distância do custo variável e ao custo fixo.

Sendo assim, uma empresa, quando inicia sua operação, terá rendimentos crescentes de escala, refletidos em custos marginais decrescentes que podem ser visualizados pelos ângulos $\beta_{1}$ e $\beta_{2}$, ilustrados na Figura 1, formados pelas tangentes ao longo do custo total.

A partir do ponto $A$, em que a empresa gera um custo médio mínimo, aumentos da produção geram custos marginais crescentes em função da ocorrência de retornos decrescentes, que podem ser vistos na Figura 1 pelos ângulos das tangentes da curva de custo total $\beta_{3}$ e $\beta_{4}$. O custo médio é a razão entre o Custo Total e a Quantidade produzida. Em termos geométricos, o ângulo formado por uma reta que parte da origem e tangencia a curva de custo total é dada pela razão entre o cateto oposto e o cateto adjacente, ou seja, Custo Total e Quantidades respectivamente (no caso da Figura 1). Assim, o menor ângulo possível de uma reta que parte da origem e tangencia a curva de Custo Total e, consequentemente o menor custo médio possível, é aquele mostrado na Figura 1 como " $\alpha$ ", ou seja tga = Custo Médio Mínimo.

Assim, a curva de custos de curto prazo pode também ser representada pelo custo médio e pelo custo marginal. Rubinfeld e Pindyck, (2002, p. 209) afirmam que "sempre que o custo marginal for inferior ao custo médio, a curva de custo médio apresentará declínio. Sempre que o custo marginal estiver acima do custo médio, a curva de custo médio apresentará elevação".

É importante, então, compreender que, na faixa em que o custo marginal estiver abaixo da curva de custo médio, cada unidade adicional de produto fará com que haja redução do custo médio até o ponto de mínimo, a partir do qual a produção de uma unidade extra de produto aumentará o custo médio.
Na decisão de produção, é importante a distinção entre custos de curto prazo e os de longo prazo. O longo prazo é caracterizado como aquele em que todos os insumos são variáveis e o de curto prazo, no qual pelo menos um insumo é fixo.

A análise das curvas auxilia as empresas no problema de minimização dos custos totais de produção. No longo prazo, a empresa tem a flexibilidade de variar a quantidade de capital de maneira que possa reduzir seus custos ou mesmo tomar decisões de expansão. Besanko e Braeutigam (2004, p. 207) afirmam que a curva de custo total de longo prazo "mostra como o custo total varia com a produção, mantendo-se constantes os preços dos insumos". Segundo os mesmos autores, no longo prazo, a empresa pode variar todos os seus insumos possibilitando que altere a proporção dos insumos para minimizar custos.

A Figura $2 \oslash$ permite vislumbrar essa relação, comparando a curva de custo total (CT) com as curvas de custo marginal $(\mathrm{CMg})$ e custo médio $(C M e)$ no ponto mínimo de custo.

Ilustra-se na Figura 2 (a) o ponto de minimização de custos no qual a linha pontilhada que parte da origem é uma tangente à curva de custo total (CT), no ponto $A$. Observe-se, também, que esse ponto está representado por A' na Figura $2(b)$, em que a curva de custo marginal $(C M g)$ é interceptada pela curva de custo médio ( $\mathrm{CMe}$ ). Besanko e Braeutigam (2004, p. 222) explicam que "a curva de custo marginal de curto prazo $\mathrm{CMg}$ intercepta a curva de custo médio de curto prazo e a curva de custo variável médio no ponto mínimo de cada curva" e que o custo marginal é igual à inclinação da curva de custo total de curto prazo.

Como podem ser observados na Figura 2 (b), os formatos das curvas de custo marginal e de custo médio são em $U$ em decorrência dos rendimentos crescentes e decrescentes de escala. Ao se imaginar uma empresa operando na região em que os custos médios são 


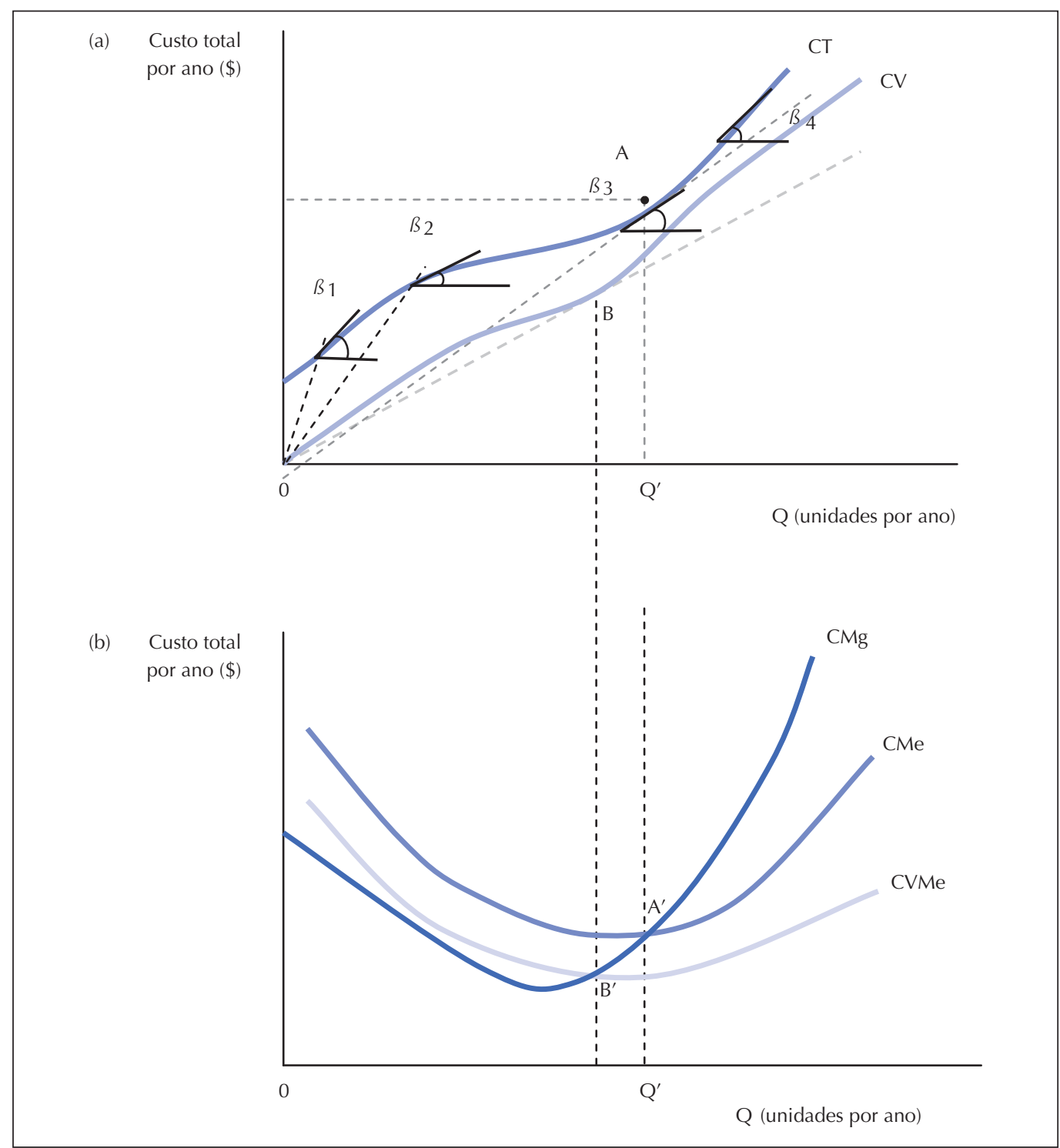

Fonte: Adaptado de Rubinfeld e Pindyck (2002, p. 210)

Figura 2 Curva de custos de curto prazo da empresa

decrescentes, ela estará obtendo ganhos de escala se estiver operando na região. A partir do ponto mínimo de custo, os custos médios serão crescentes, não obtendo, assim, os ganhos de escala.

Outra forma de pensar essa relação é se o custo marginal é o acréscimo no custo total quando $Q$ varia uma unidade, então, se o custo médio se reduz é porque o custo marginal é menor que o custo médio e se o custo médio aumenta é porque o custo marginal é maior que o custo médio. Logo, quando o custo médio é igual ao custo marginal o custo médio será mínimo. Assim, as duas curvas se cruzam onde a curva de custo médio atinge o ponto mínimo (WALSH; STIGLITZ, 2003).

A curva de custo total de longo prazo é semelhante à apresentada na Figura 2, exceto que parte da origem, visto que todos os custos são variáveis, portanto elimina-se a 
possibilidade de custo fixo. Da mesma forma, podem-se construir as curvas de custo médio de longo prazo e custo marginal de longo prazo a partir do custo total.

Analisando a relação entre a curva de custos do curto prazo com a curva de custos de longo prazo, Besanko e Braeutigam (2004, p. 220) explicam que, pelo fato de a empresa estar "livre para variar a quantidade de capital no longo prazo ela pode incorrer em custos totais inferiores aos que enfrenta quando o capital está fixo". Assim sendo, no curto prazo a empresa não pode ajustar a quantidade de capital livremente.

Dessa forma, em cada ponto da curva de custo total de longo prazo, tem-se uma planta para operar no curto prazo. Assim até o nível de produção Qn plantas maiores terão custos unitários menores, ou seja, plantas maiores obtêm economias de escala. O ponto $Q n$ representa a planta com menor custo unitário possível. Assim, para qualquer quantidade maior que $Q n$ a empresa passará a incorrer em deseconomias de escala.

\subsection{Economia de Escala}

A economia de escala ou rendimentos de escala pode explicar porque algumas empresas são mais lucrativas do que outras. Por meio da economia de escala é possível maximizar os lucros à medida que a quantidade produzida aumenta.

Alguns processos tornam-se mais produtivos em grande escala do que em pequenas por proporcionarem reduções nos custos médios de produção, em decorrências dos volumes produzidos não aumentarem os custos fixos. Isso pode ser observado quando todos os insumos são duplicados e o custo aumenta em proporção menor que o dos insumos. Para Besanko e Braeutigam (2004, p. 214), economia de escala ocorre quando "o custo médio diminui à medida que a produção aumenta”.

Em grandes indústrias de produção em massa, de acordo com Hogendorn (1975), os custos unitários são relativamente baixos apenas numa grande quantidade produzida. Sendo assim, as empresas que conseguirem um aumento no volume de produção sem aumentarem os seus custos fixos, terão ganhos de economia de escala. O ganho de escala demonstra o aumento percentual na produção quando a empresa aumenta o volume dos insumos de produção em uma dada porcentagem, auferindo vantagem competitiva pela redução de custos médios proporcionada.

Já o oposto ocorre com as deseconomias de escala ou rendimentos decrescentes de escala, nos quais, de acordo com Wessels (2002, p. 89), ao se "aumentar todos os insumos em $\mathrm{x} \%$, a produção subir menos do que $\mathrm{x} \%$ ” reduzindo a proporção entre a produção e os insumos. Besanko e Braeutigam (2004, p. 214) esclarecem que em deseconomias de escala "o custo médio aumenta à medida que o produto aumenta".

Pensando em termos de função de custo de longo prazo, que é representada graficamente por uma parábola, Garófalo e Carvalho (1990) esclarecem que podem ser observadas em gráficos de custo médio de longo prazo as economias e deseconomias de escala, evidenciando setores de expansão da produção a custos crescentes à direita do ponto de mínimo e decrescentes à esquerda do ponto.

Um dos motivos para a existência de rendimentos crescentes de escala, para Wessels (2002), é que muitos processos de produção exigem uma grande escala para funcionar eficazmente e que uma das principais razões para deseconomias de escala é que, com o crescimento das empresas se torna mais difícil de serem administradas. $\mathrm{O}$ autor, também, explica que a divisão de trabalho, ou seja, a especialização dos empregados, na execução de uma ou poucas tarefas, possibilita ganhos de escalas na produção.

Thompson e Formby (1998, p. 148) chamam a atenção para economias de escalas advindas de uma planta maior estar relacionada às economias resultantes da produção em massa, por permitirem "maiores subdivisões 
no processo de produção e maior especialização no uso de insumos e fatores de produção (trabalho, capital e supervisão)".

Dessa forma, para que se possa verificar a existência ou não de economias de escala é importante a determinação da função de custos, cujos conceitos são determinados na seção seguinte.

\subsection{Função de Custos}

Buscando entender a relação dos custos médios com a quantidade produzida, apresenta-se a função de custos visando a embasar os conceitos e condições para se estimar a função de custos do setor de telefonia móvel brasileiro.

A função custo total é "uma relação matemática que mostra como o custo total varia com os fatores que influenciam o custo total" (BESANKO; BRAEUTIGAM, 2004, p. 231), permitindo a determinação de como o custo varia em relação às quantidades de insumos aplicados na produção e o nível de produção obtido.

Para Klein (1978, p. 132), a função de custo "sintetiza muitos fatores que compõem a teoria em termos de função de produção e condições de produtividade marginal". O autor esclarece que a função de produção é uma relação de muitos fatores ao passo que a função de custo é "uma relação bivariada, geralmente, que associa o produto ao custo total".

Sendo assim, entende-se que o objetivo ao estimar a função de custo, é encontrar uma relação entre as variáveis e poder usá-las para previsão. Para a estimação da função custo, Rubinfeld e Pindyck (2002, p. 235) esclarecem que a função de custo pode ser obtida por intermédio de diversas relações funcionais, como, por exemplo, relação linear, re- gressão por mínimos quadrados, função cúbica e função translog.

A estimação pelo método de mínimos quadrados, segundo Kmenta (1978, p. 223), "envolve a minimização da soma dos desvios ao quadrado, dos valores observados a partir da média. Isto é, temos de encontrar o valor da média que faça a soma exigida tão pequena quanto possível".

Como as curvas de custo marginal e de custo médio de curto ou de longo prazo têm formato $U$, Rubinfeld e Pindyck (2002) recomendam uma função cúbica para a obtenção da função de custo total. Garófalo e Carvalho (1990) afirmam que a função cúbica é a mais comumente empregada para a representação gráfica do custo total.

Sendo assim, a função cúbica pode ser representada, de acordo com Gujarati (2000, p. 209) pela equação polinomial de terceiro grau:

$Y=\beta_{0}+\beta_{1} X+\beta_{2} X^{2}+\beta_{3} X^{3}+u i$

em que:

$Y=$ custo total a ser estimado;

$\beta_{0}=$ é o custo fixo;

$\beta_{1} X+\beta_{2} X^{2}{ }_{i}+\beta_{3} X^{3}$ custo variável;

$\beta_{1}, \beta_{2^{2}} \beta_{3}=$ parâmetros que medem a sensibilidade do custo variável para uma variação de uma unidade na produção;

$X=$ quantidade produzida;

$u_{i}=$ erro aleatório que inclui a influência de outros elementos explicativos dos custos que não estão computados no modelo.

Estima-se que os sinais esperados dos coeficientes dos parâmetros $\beta_{0}, \beta_{1}$ e $\beta_{3}$ sejam maiores que zero e que do parâmetro $\beta_{2}$ seja menor que zero (GUJARATI, 2000).

\section{PROCEDIMENTOS METODOLÓGICOS}

Esta pesquisa é de natureza aplicada, visando a gerar conhecimento prático à solução de problemas específicos na área econômica.
Do ponto de vista dos objetivos, o estudo é classificado como descritivo. No que se refere à abordagem do problema, a pesquisa é quan- 
titativa. Do ponto de vista dos procedimentos técnicos, é do tipo documental (GIL, 1999; SILVA; MENEZES, 2001).

Foram pesquisadas todas as operadoras de telefonia móvel brasileiras, do período de 1998 até 2005 . No início deste estudo, após as privatizações do setor, existiam 38 operadoras. Ao longo do período analisado 11 operadoras sofreram processos de incorporações societárias, bem como 4 novas empresas entraram em operação. Dessa forma, ao final de 2005, o setor de telefonia móvel contava com 31 operadoras, sendo que se obtiveram informações de 30 empresas.

Para estimação da função de custo do setor foi aplicada a análise de regressão polinomial de terceiro grau, utilizando o software estatístico Eviews for Windows, versão 5.1, pelo método de mínimos quadrados, que é o mais utilizado para estimar a reta de regressão. Esse consiste em determinar a reta de melhor ajuste minimizando a soma dos erros (GUJARATI, 2000). A técnica utilizada foi a de dados em painel com efeitos fixos, tendo como base os pressupostos sobre o termo de erro e sobre as especificações do modelo.

A estimação com dados em painel sugere a existência de características diferenciadoras dos indivíduos mediante a revelação da heterogeneidade individual. Nos modelos de efeitos fixos, os coeficientes podem variar de indivíduo para indivíduo ou no tempo, ainda que permaneçam como constantes fixas (MARQUES, 2000).

A equação inicial testada, conforme apresentada na fundamentação teórica, foi:

$Y_{i t}=\beta_{0 i t}+\beta_{1} X_{i t}+\beta_{2} X_{i t}^{2}+\beta_{3} X_{i t+}^{3} u_{i t}$

em que:

$Y_{i t}=$ custo total a ser estimado da empresa i, no tempo t;

$\beta_{\text {oit }}=$ é o custo fixo;

$\beta_{1} X_{i t}+\beta_{2} X_{i t}^{2}+\beta_{3} X_{i t}^{3}=$ custo variável;

$\beta_{1}, \beta_{2}, \beta_{3}=$ parâmetros que medem a sensibi- lidade do custo variável para uma variação de uma unidade na produção;

$X_{i t}=$ quantidade produzida pela empresa i no período t;

$u_{i t}=$ erro aleatório que inclui a influência de outros elementos explicativos dos custos que não estão computados no modelo i, no período t.

Quanto aos sinais dos coeficientes da regressão, espera-se, de acordo com a teoria, que o $\beta_{0} \beta_{1}$ e $\beta_{3}$ sejam positivos e o coeficiente $\beta_{2}$ seja negativo.

Foram aplicados os testes Estatística- $t$ e Estatística- $F$, com o objetivo de avaliar relações entre as variáveis dependente e independente do modelo e o Teste de Durbin-Watson para verificar se os erros (resíduos) do modelo gerado são autocorrelacionados. Para verificação da normalidade da distribuição dos resíduos foi testada a Estatística de Jarque-Bera (HILL; GRIFFITHS; JUDGE, 2003; RUBINFELD; PINDYCK, 1998). O nível de $5 \%$ de significância foi determinado para os resultados obtidos.

Os valores de $Y$, variável dependente, foram os somatórios trimestrais de: (a) custos dos serviços prestados; (b) despesas administrativas e (c) despesas com vendas. Esses foram obtidos por meio das demonstrações contábeis publicadas pelas empresas, disponíveis no site da Comissão de Valores Mobiliários (CVM), no site da Economática, em jornais nacionais, bem como nos sites das operadoras celulares do Brasil, do período de 31 de março de 1998 a 31 de dezembro de 2005.

Como valores de $X$, variável independente da função, foram adotados os números de clientes (acessos móveis em operação), pela indisponibilidade das informações do tráfego gerado. Em estudos similares no setor, em outros países, o número de clientes foi adotado como quantidade produzida (valor de $\mathrm{x}$ ). Esses foram obtidos nos relatórios de administração que acompanham os balanços patrimoniais publicados trimestralmente pelas operadoras, bem como na Agência Na- 
cional de Telecomunicações (ANATEL), que forneceu os acessos consolidados mensais do setor para o período analisado. Nos casos de indisponibilidade dessa informação pelas operadoras, foram utilizadas as informações divulgadas pela Teleco (2006).

Os dados coletados foram agrupados de acordo com a sua natureza, em ordem cronológica, numa série trimestral no período analisado para o conjunto de empresas pesquisadas, organizados em tabelas e gráficos. Foi procedida à harmonização dos dados monetários extraídos das demonstrações contábeis para torná-las homogêneas no tempo, ou seja, foram deflacionados pelo Índice Geral de Preços do Mercado (IGPM), apurados pela Fundação Getúlio Vargas, na data-base de dezembro de 2005.

\section{ANÁLISE DA ECONOMIA DE ESCALA NO SETOR}

Essa seção apresenta os resultados referentes à modelagem e estimação da função de custos para o setor de telefonia móvel, com fins de analisar a existência ou não de economia de escala no setor, bem como verificar o ponto da curva de custo médio em que estavam localizadas as operadoras do setor em dezembro de 2005.

\subsection{Estimação da Função de Custo Total}

A função de custo total para o setor foi estimada por uma equação polinomial, conforme apresentado no capítulo de metodologia. Inicialmente, apresentam-se, na Tabela $1 \mathbf{0}$, algumas medidas estatísticas básicas das variáveis consideradas no modelo de regressão.
A média da variável $C T$ foi de $\mathrm{R} \$ 205,2$ milhões, tendo tido como valor máximo $\mathrm{R} \$$ 1,26 bilhões e mínimo de $\mathrm{R} \$ 1,7$ milhão. $\mathrm{O}$ número médio de clientes $(Q)$ foi de 1,26 milhão, enquanto o número máximo foi de 10,47 milhões.

Tendo em vista algumas características diferenciadoras, como, por exemplo, quantidade de clientes e densidade populacional das áreas de atuação entre as operadoras de telefonia móvel brasileiras, a média dos valores do custo total apresenta valores extremos. O valor médio mínimo da operadora Telaima foi de $\mathrm{R} \$ 4,4$ milhões e o valor máximo da operadora Telesp de R 894 milhões. Das 30 operadoras incluídas na amostra, 76,7\% apresentaram um valor médio de custo total

Tabela 1 Estatística descritiva da amostra

\begin{tabular}{l|c|c}
\hline ESTATístICA DESCRITIVA & C - CUSTO TOTAL $-\mathbf{R} \$$ mil & $\mathbf{Q}$ - QUANTIDADE - mil \\
\hline Média & 205.207 & 1.259 \\
\hline Máximo & 1.260 .061 & 10.476 \\
\hline Mínimo & 1.728 & 6 \\
\hline Desvio-Padrão & 221.465 & 1.699 \\
\hline Assimetria & 1,97 & 2,53 \\
\hline Curtose & 7,44 & 10,74 \\
\hline Observações & 691 & 691 \\
\hline Empresas (Cross sections) & 30 & 30 \\
\hline
\end{tabular}

$\mathrm{Q}=$ número de clientes 
até R\$ 300 milhões, o que explica a média de custo total de R\$205 milhões.

Apresenta-se, então, na Tabela $2 \boldsymbol{0}$, a função do custo total estimada para o setor de telefonia móvel brasileiro para o período pósprivatizações, em que a variável dependente é custo total e a variável independente é a quantidade de clientes.

A equação do custo total estimada apresentou os sinais dos coeficientes esperados, previstos nos requisitos teóricos de retornos crescentes e decrescentes, ou seja, $\beta_{1}$ e $\beta_{3}$ maiores que zero e $\beta_{2}<0$.

A regressão estimada foi significativa a $1 \%$ nos testes $F$ e $t$, evidenciando que os coeficientes dos parâmetros $\beta_{0,} \beta_{1} \beta_{2}$ e $\beta_{3}$ são estatisticamente diferentes de zero. Assim, junto com a análise da probabilidade, que representa um índice decrescente da confiabilidade de um resultado, que é menor que o nível de significância escolhido, entende-se que os parâmetros das variáveis são representativos para a estimação da função de custo. Nessa, a variação do número de clientes $(Q)$ explica a variação do custo total $(C T)$ do setor de telefonia móvel brasileiro no período pós-privatizações.

O R ${ }^{2}$ ajustado é um coeficiente de determinação que diz o quão bem a curva de regressão estimada se ajustou aos dados. Obteve-se um $\mathrm{R}^{2}$ ajustado de 0,9091, ou seja, 90,91\%: um elevado nível de explicação.

O resultado da estatística de Durbin-Watson foi de 0,8887 , que é próximo de zero, o que revela que os erros possuem autocorrelação positiva, de primeira ordem a um coeficiente de autocorrelação (AC) de 0,517. Para correção da autocorrelação identificada, como sugere a literatura, foi aplicado no modelo um termo autoregressivo (AR), na equação testada inicialmente, entretanto persistiu a autocorrelação.

Acredita-se que os estimadores obtidos atendem à proposta do trabalho. Isso porque, segundo Gujarati (2000), os estimadores de regressões com autocorrelação são não viesados, mas ineficientes para previsões e o objeto da pesquisa é estimar a curva de custo conforme apresentada na fundamentação teórica e não para realizar previsões ou para testar hipóteses, além de o modelo gerado se ajustar bem aos dados.

Os resíduos da equação estimada apresentaram dispersão em torno de zero, sem um formato específico dos resíduos sugerindo não existir problemas de heteroscedasticidade (GUJARATI, 2000). Para verificar se existe normalidade nos resíduos, foi aplicado o teste de Jarque-Bera. Esse revelou que não há normalidade nessa distribuição $(6897,83)$.

Tabela 2 Equação estimada do custo total do setor de telefonia móvel brasileiro

\begin{tabular}{|c|c|c|c|c|c|}
\hline PARÂMETRO & $\begin{array}{c}\text { VARIÁVEL } \\
\text { INDEPENDENTE }\end{array}$ & COEFICIENTE & $\begin{array}{l}\text { DESVIO } \\
\text { PADRÃO }\end{array}$ & $\begin{array}{c}\text { ESTATÍSTICA } \\
-t\end{array}$ & PROBABILIDADE \\
\hline$\beta_{0}$ & Constante & $130.708,4$ & 6099,130 & 21,430660 & 0,0000 \\
\hline$\beta_{1}$ & Q & 99,47651 & 10,22116 & 9,732412 & 0,0000 \\
\hline$B_{2}$ & $Q^{2}$ & $-0,016845$ & 0,003031 & $-5,557362$ & 0,0000 \\
\hline$B_{3}$ & $Q^{3}$ & $9,70 \mathrm{E}-07$ & $2,24 \mathrm{E}-07$ & 4,325305 & 0,0000 \\
\hline \multicolumn{6}{|c|}{ ESTATÍSTICAS } \\
\hline R-quadrado & 0,913405 & & & Estatística- $F$ & 216,8948 \\
\hline $\begin{array}{l}\text { R-quadrado } \\
\text { ajustado }\end{array}$ & 0,909194 & $\begin{array}{l}\text { Teste Durbin- } \\
\text { Watson }\end{array}$ & 0,888778 & $\begin{array}{l}\text { Probabilidade } \\
\text { Estatística-F }\end{array}$ & 0,0000 \\
\hline
\end{tabular}


O valor da assimetria para a equação estimada do custo total foi de $-0,164$, com um indicador de curtose de 18,47, o que confirma a não normalidade, conforme as medidas de assimetria $=0$ e curtose $=3$, previstas para esse teste, sendo essa a mais forte deficiência dos dados analisados, uma vez que a teoria clássica pressupõe $u i \sim N\left(0, \sigma^{2}\right)$.

A normalidade dos erros do modelo de regressão também não é satisfeita, devido à presença de muitos outliers (observações que apresentam um grande afastamento das observações restantes da amostra) no setor de telefonia móvel. Entretanto, é comum a presença de outliers em dados econômicos, haja vista a variabilidade inerente dos elementos da população. No entanto, na prática, tais violações não comprometem as análises, pois contêm informações relevantes sobre características subjacentes aos dados e poderão ser decisivas no conhecimento da população à qual pertence a amostra em estudo (FIGUEIRA, 1998). Os principais outliers identificados a partir dos resíduos individuais foram Telesp Celular S.A. e a Tele Sudeste Celular Participações S.A.

Assim sendo, substituindo os coeficientes estimados da regressão na equação, tem-se a função do custo total para o setor.
$C T=130.708,38+99,47 * Q-$ $0,016 * Q^{2}+9,70 \times 10^{-7 *} Q^{3}$

Dessa forma, o valor de $\beta_{0}=130.708,38$ representa o custo fixo total do setor de telefonia móvel. Os valores de $\beta_{1}=99,47, \beta_{2}=$ $-0,016$ e $\beta_{3}=9,70 \times 10^{-7}$ multiplicado pelas quantidades produzidas $(Q)$ evidenciam o custo variável do setor, ou seja, quanto varia o custo total do setor quando aumenta um cliente de telefonia móvel.

\subsection{Curvas de Custo do Setor}

A partir da função de custo total estimada para o setor de telefonia móvel, apuraram-se as curvas de custo médio e marginal desse. Representando a curva de custo total do setor de telefonia móvel a partir da função de custo estimada, obteve-se o formato visualizado na Figura 30.

O formato da curva de custo total, observado na Figura 3, decorre da relação dos rendimentos crescentes e decrescentes, conforme referenciado na fundamentação teórica, pois os custos variáveis do setor aumentam com a quantidade produzida à taxa decrescente até certo nível de produção.

Essa situação contribuiu para atrair novas empresas para o setor quando da abertura das

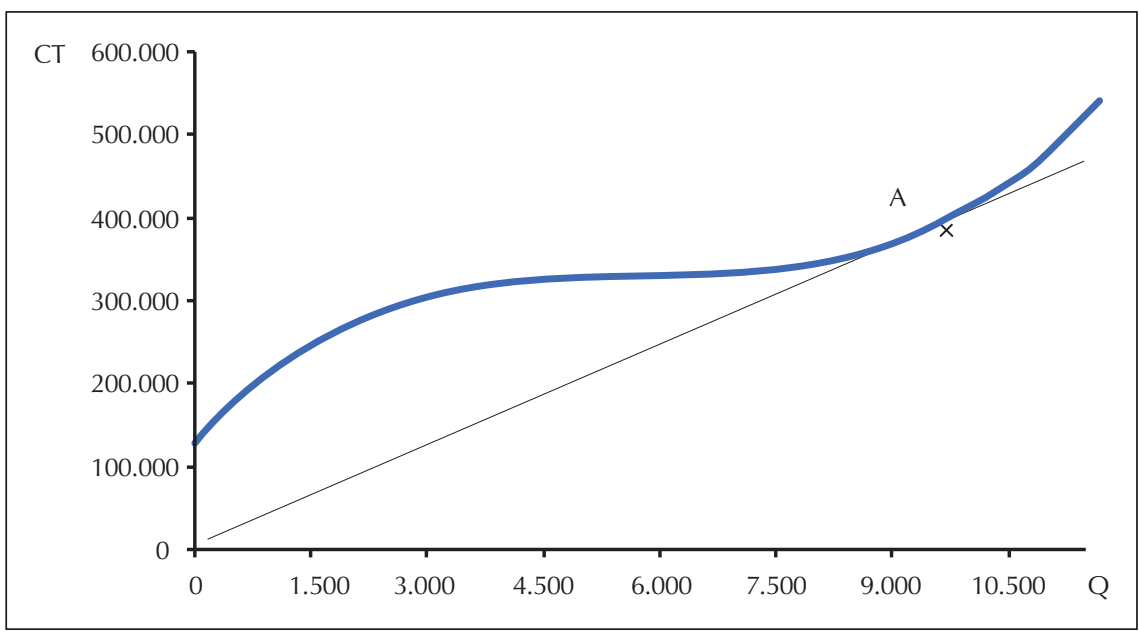

Figura 3 Curva estimada de custo total do setor de telefonia móvel brasileiro 
Bandas D e E. Entretanto, à medida que a produção aumentou, chegou a um ponto a partir do qual o custo total cresceu mais rapidamente (Ponto A), representado pela tangente à curva de custo total $(C T)$ da linha pontilhada que parte da origem. Para a identificação dessa quantidade de maximização dos lucros do setor, procedeu-se à análise das curvas de custo médio e custo marginal. Partindo-se da equação estimada do custo total para o setor de telefonia móvel, calcularam-se as funções de custo médio e custo marginal. A equação de custo médio foi obtida pela divisão da equação de custo total (CT) pela quantidade Q. Assim obteve-se a equação:

\section{$\mathrm{CMe}=$}

$\frac{130.708,38+99,47 * Q-0,016^{*} Q^{2}+9,70 \times 10^{-7 *} Q^{3}}{Q}$

Partindo-se da equação estimada do custo total para o setor de telefonia móvel, obtevese a curva de custo marginal pela derivação da equação de custo total pela quantidade $Q$. A derivada da função de custo total indica a taxa de variação do custo total quando varia a quantidade, ou seja, o custo marginal. Dessa forma, esse custo reflete a inclinação da tangente à função do custo total no ponto indicado. A função estimada para o custo marginal do setor foi:

$C M g=99,47650949-0,03369 * Q+$ $0,0000029 * Q^{2}$

Derivando-se a função de custo médio e igualando-a a zero, encontrou-se a quantidade de 9,44 milhões de clientes, que é a quantidade que gera o custo médio mínimo. Assim, procedeu-se à derivação da função.

Apresenta-se a análise realizada da existência de economia de escala no setor de telefonia móvel brasileiro, por meio da função de custo.

Conforme abordado na fundamentação teórica, ocorre economia de escala quando a expansão da capacidade de produção de uma empresa causa um aumento proporcionalmente menor dos custos totais de produção do que os do produto. $\mathrm{O}$ que resulta em custos médios de produção decrescentes.

Para tanto, por intermédio das funções de custo marginal e de custo médio, obtidas a partir da estimação da função do custo total, este estudo visou a identificar o ponto mínimo de custo da produção da indústria de telefonia celular brasileira, a partir do qual aumentos da produção sejam realizados com retornos decrescentes que levem a custos marginais crescentes.

A Figura $4 \bullet$, apresenta as curvas de CMe e $C M g$ e, no ponto em que as curvas de CMe e $C M g$ se encontram, tem-se o ponto de mínimo do custo médio, conforme justificado na fundamentação teórica.

O formato da curva de custo marginal em $U$ denota a existência de rendimentos crescentes e decrescentes na função de produção do setor de telefonia móvel no Brasil, no período analisado. Assim, observando a Figura 4, a curva de custo marginal estimada para o setor de telefonia móvel indicou o ponto de inflexão em torno da quantidade de 6.000 mil. Logo, até 6 milhões de clientes, existem retornos crescentes de produtividade. A partir desse ponto, um aumento na produção do setor (mais clientes) gera redução da produtividade marginal.

A curva de custo médio mostra que, com o aumento da produção de telefonia móvel, aqui medida pelo aumento do número de clientes na planta das operadoras, os custos fixos foram diluídos a cada unidade adicional de clientes. Assim, o CMe reduziu com o aumento na quantidade de clientes até uma quantidade $X$ e depois passou a aumentar.

Na parte declinante da curva de CMe existe economia de escala nas plantas do setor de telefonia móvel, pois o custo médio declinou à medida que aumentou o tamanho da planta, em razão da economia de escala alcançada pelas operadoras de telefonia móvel. 


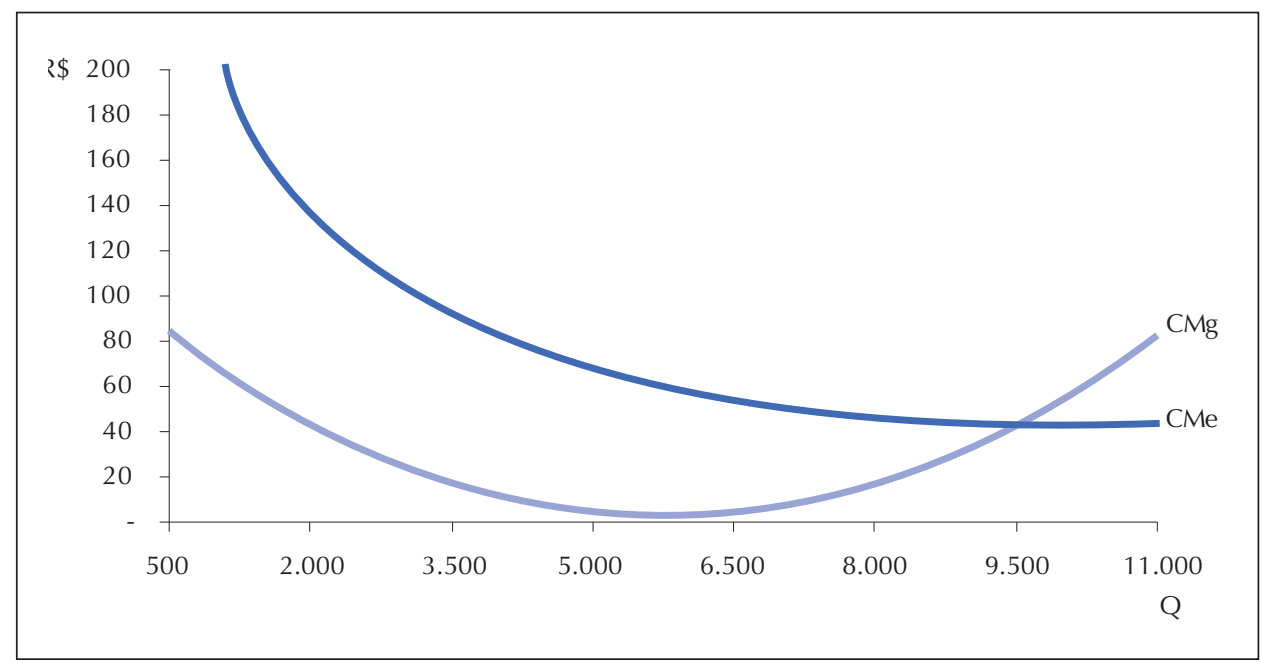

Figura 4 Curvas de custo médio e marginal do setor de telefonia móvel brasileiro

Como essa curva é a do setor, então, empresas com um número menor de 9,44 milhões de clientes não estavam se beneficiando dos ganhos de escala. Empresas operando com o esse número de clientes apresentam a melhor planta, dada a estrutura de custo e tecnologia existente, pois podem operar com o menor custo mínimo. Já as empresas com um número maior que esse passaram a operar em uma região com perda de economia devido à saturação de um dos insumos e das possibilidades tecnológicas atuais.

A quantidade estimada do ponto em que a curva de $C M g$ encontra a curva de $C M e$ foi 9,44 milhões de clientes. Sendo assim, até esse ponto os acréscimos marginais do custo foram inferiores ao custo médio. A partir de 9,44 milhões de clientes, o $\mathrm{Cmg}>\mathrm{Cme}$ de forma que o custo total do setor de telefonia móvel brasileiro crescesse.

\subsection{Economia de Escala}

Dado o número de clientes das operadoras celulares no Brasil ao final de 2005, a maioria encontrava-se localizada na região de rendimentos crescentes. O posicionamento das operadoras, em relação à função de custo médio estimada para o setor, está demonstrado na Figura $5 \bullet$.
A disposição das empresas obedeceu à quantidade de clientes e ao valor do custo médio de cada operadora em dezembro de 2005. A quantidade estimada que minimiza o custo do setor está representada na Figura 5 pelo ponto $\mathrm{A}$, ou seja, 9,44 milhões de clientes.

As operadoras Americel S.A., Celular CRT S.A., Global Telecom S.A., Telemig Celular S.A., Telet S.A., Tim Nordeste Telecomunicações S.A. e Tim Sul S.A. encontramse concentradas em torno da quantidade de 3 milhões de clientes e custo médio no intervalo de R\$ 80 a R\$90, ao final de 2005 . Mesmo que essas operadoras dupliquem a produção, ainda assim, estarão atuando na região de rendimentos decrescentes de escala. Portanto, para que as operadoras possam intensificar a capacidade da planta instalada, precisam continuar expandindo a produção, ou seja, a base de clientes, para usufruírem os ganhos de escala. As decisões de expansão da indústria implicam a escolha de diferentes quantidades de fatores e insumos a utilizar.

As operadoras Telesp Celular S.A. e TNL PCS S.A. (Oi) encontravam-se, ao final de 2005, com 10,47 e 10,3 milhões de clientes, respectivamente, ou seja, com um número superior ao ponto de mínimo da curva de 


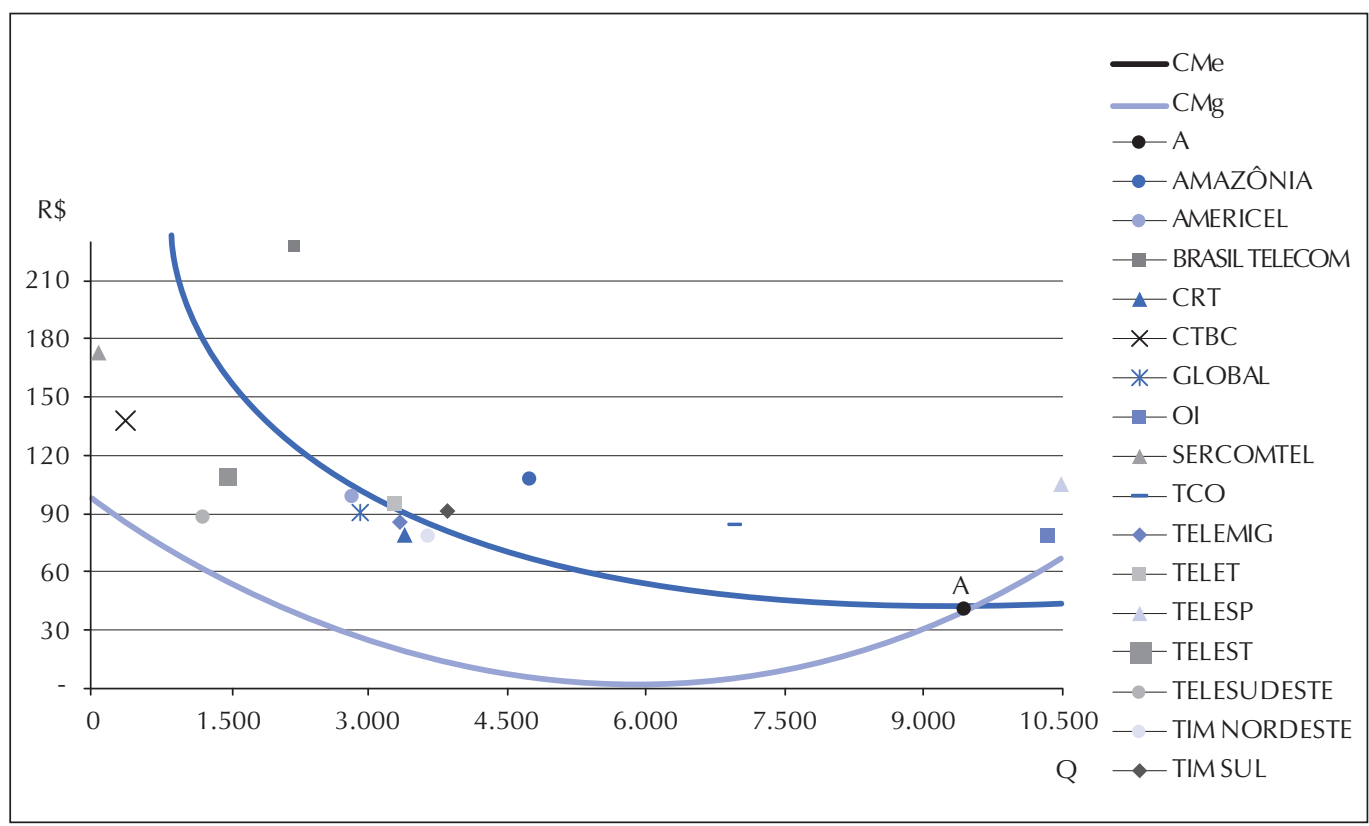

Figura 5 Posicionamento das empresas de telefonia móvel brasileira em dezembro de 2005

custo médio estimada do setor. Portanto, já passaram da planta ótima de produção para o setor.

A questão da economia de escala pode ter motivado os processos de aquisições e formação de alianças estratégicas após as privatizações do setor. Das 38 operadoras de telefonia celular, existentes no início do estudo, 11 sofreram processos de incorporação ao longo do período analisado, em busca de alguma vantagem competitiva proporcionada pela economia de escala, redução de custos, desenvolvimento conjunto de novos produtos, entre outras.

A questão levantada por este estudo visa a saber se o número de empresas de telefonia móvel é adequado à demanda do mercado. Assim, dado que, em dezembro de 2005, o parque móvel brasileiro possuía $86,2 \mathrm{mi}$ lhões de clientes com 31 empresas em operação, dividiu-se essa quantidade de clientes pelo número de empresas. A quantidade média apurada foi bem aquém do ponto de mínimo estimado, totalizando 2,78 milhões de clientes.
Essa situação evidencia que o setor encontrava-se com excessivo número de empresas, portanto, com uma grande perda de economia de escala. Para que as empresas estivessem usufruindo os benefícios dos ganhos de escala, deveriam estar com a produção próxima do ponto de minimização de custo.

Dividindo-se o total de clientes, ao final de 2005 (86,2 milhões), pela quantidade de minimização de custos (9,44 milhões), temse que o número de empresas deveria ser aproximadamente de dez.

Em Portugal, Carreira (1999) estimou economia de escala para a quantidade de 3,5 milhões de assinantes, com duas empresas atuantes no setor, sinalizando que o mercado Português não suportaria o ingresso de mais uma operadora, devendo permanecer duopolista.

Os três principais grupos controladores, VIVO, TIM e CLARO, detinham de forma consolidada $79,6 \%$ do parque de clientes do setor, ao final de 2005. O Grupo VIVO encontrava-se com 29,8 milhões de clientes. O Grupo TIM finalizou o ano com 20,17 milhões 
de clientes e o Grupo CLARO, com 18,65 milhões de clientes (TELECO, 2006). Em quarto lugar, estava o Grupo TELEMAR (OI) com 10,34 milhões de clientes, perfazendo $91,6 \%$ dos clientes totais do setor. As demais empresas representavam somente $0,08 \%$ da base de clientes do setor ao final do ano de 2005.
Dessa forma, levando em consideração as pressões da concorrência e a organização do mercado por grupos controladores, as operadoras tendem a continuar os processos de fusões e incorporações. Isso para que consolidem suas posições de maneira sustentável no mercado e obtenham os benefícios de escala.

\section{CONCLUSÃO}

A análise pela função de custo consistiu em determinar a existência de economia de escala mediante estudo das curvas de custos do setor. Pela estimação da função de custos, verificou-se a existência de economias de escala até 9,44 milhões de clientes - ponto de mínimo do custo médio do setor - e deseconomias de escala a partir desse ponto. Ao final do período analisado, a maioria das operadoras encontrava-se localizada na curva de custo médio estimada para o setor, produzindo em média a quantidade de 2,78 milhões de clientes. Nesse nível produtivo, as operadoras não estavam usufruindo os benefícios dos ganhos de escala. Assim sendo, as operadoras necessitam aumentar as quantidades produzidas, ou seja, o número de clientes, até o ponto de minimização de custos, visando a se beneficiarem da economia de escala.

Analisando o número de empresas do setor, que, ao final do ano de 2005 , era de 31 operadoras, apurou-se que o mercado se encontrava, com um número excessivo de empresas à demanda do mercado. $\mathrm{O}$ estudo, também, revelou que esse número deveria ser em torno de dez operadoras, para que as empresas do setor pudessem usufruir os benefícios da economia de escala gerada no processo produtivo.

Do ponto de vista da gestão estratégica de custos, operar próximo do ponto de mínimo dos custos, pode representar uma questão vital à sobrevivência das empresas nesse ambiente altamente competitivo, visto a busca constante do diferencial tecnológico repre- sentar um fator determinante à competitividade do setor.

Dadas as transformações estruturais por que passou o setor de telefonia móvel no Brasil, motivadas pela implementação do modelo adotado para a privatização do setor, foi desenvolvida uma complexa estrutura oligopolista. Como as decisões de uma empresa, nesse mercado, afetam significativamente os lucros dos concorrentes, conforme apresentado na fundamentação teórica, a maximização dos lucros torna-se muito difícil de ser estabelecida individualmente.

Essa característica do oligopólio, provavelmente, colaborou para a continuidade dos processos de fusões e incorporações entre as empresas do setor, pois se observou, adicionalmente, ao período de escopo deste trabalho, que os Grupos VIVO, TIM e CLARO realizaram importantes incorporações societárias ao longo de 2006.

Com isso, o número de empresas, que era de 38 operadoras ao início das privatizações do setor, após as incorporações ocorridas ao longo do período, reduziu-se para 9 operadoras ao final de 2006, reforçando a estrutura de mercado de oligopólio do modelo brasileiro de telecomunicações, evidenciando que o problema de pesquisa objeto deste trabalho era pertinente.

Assim sendo, acredita-se que as empresas estavam em busca da vantagem competitiva de custos, proporcionada pela economia de escala da produção em maior volume, bem como da ampliação das suas áreas de coberturas dos serviços ofertados aos consumido- 
res, entre outras razões, de forma a obterem ou ampliarem a participação no mercado.

Finalmente, sugere-se, em futuras pesquisas no setor de telecomunicações móveis, investigar se a adoção de diferentes padrões tecnológicos, bem como o grau de inovação tecnológica implementados pelas operadoras, contribuiu diferentemente para a formação das margens de lucros das operadoras brasileiras após as privatizações, permitindo compreender melhor a relação mercado consumidor e estruturas de custos.

\section{Referências}

BESANKO, D.; BRAEUTIGAM, R. R. Microeconomia uma abordagem completa. Rio de Janeiro: LTC, 2004. CARREIRA, C. M. G. A Abertura do mercado de telecomunicações celulares ao terceiro operador: uma decisão racional? Grupo de Estudos Monetários e Financeiros (GEMF) da Faculdade de Economia da Universidade de Coimbra, Coimbra, n. 8. 1999. COMISSÃO DE VALORES MOBILIÁRIOS. Informações sobre companhias abertas: sistema de divulgação externa. Disponível em: <http://www.cvm.gov.br/indexpo.asp >. Acesso em: diversos em 2006.

FIGUEIRA, M. M. C. Identificação de outliers. Revista Millenium Online, Viseu, n. 12. Out. 1998. Disponível em: <http://www.ipv.pt/millenium/arq12.htm>. Acesso em: 05 fev. 2007.

GARÓFALO, G. L.; CARVALHO, L. C. P. Teoria econômica. 2. ed. São Paulo: Atlas, 1990.

GIL, A. C. Métodos e técnicas de pesquisa social. São Paulo: Atlas, 1999.

GUJARATI, D. N. Econometria básica. São Paulo: Makron Books, 2000.

HILL, R. C.; GRIFFITHS, W. E.; JUDGE, G. G. Econometria. 2. ed. Tradução Alfredo Alves de Farias. São Paulo: Saraiva, 2003.

HOGENDORN, J. S. O mercado na economia moderna: uma introdução à microeconomia. Rio de Janeiro: Zahar, 1975.

KLEIN, L. R. Introdução à econometria. São Paulo: Atlas, 1978.

KMENTA, J. Elementos de econometria. São Paulo: Atlas, 1978.

MARQUES, L. D. Modelos dinâmicos com dados em painel: revisão de literatura. Centro de Estudos
Macroeconômicos e Previsão (CEMPRE) da Faculdade de Economia do Porto. Porto, Out. 2000.

NEVES, M. S. O setor de telecomunicações. In: BNDES 50 Anos - Histórias Setoriais. Rio de Janeiro: BNDES, 2002. Disponível em: <http://www.bndes.gov.br/ conhecimento/livro_setorial/setorial13.pdf $>$. Acesso em: 23 nov. 2005.

PESSINI, J. E. Telecomunicações. In: Economia do Ministério das Relações Exteriores. Disponível em: <http:/ www.mre.gov.br/cdbrasil/Itamaraty/web/port/economia/ teleco/apresent/index.htm>. Acesso em: 27 dez. 2005. RODRIGUES, D. A. Os investimentos no Brasil nos anos 90: cenários setorial e regional. Revista do BNDES, v. 7 n. 13, p. 107-138. Rio de Janeiro, Junho, 2000. RUBINFELD, D. L.; PINDYCK, R. S. Economic models and economic forecast. 4. ed. Mcgraw-Hill Professi, 1998. Microeconomia. 5. ed. São Paulo: Person, 2002.

SILVA, E. L.; MENEZES, E. M. Metodologia da pesquisa e elaboração de dissertação. Florianópolis, 2001. Disponível em: <http:/www.ufsc.br>. Acesso em: 18 mar. 2005. TELECO, Informações em telecomunicações. Telefonia celular. Disponível em: <http:www.teleco.com.br/ Operadoras/>. Acesso em: 18 ago. 2006.

THOMPSON Jr., A. A.; FORMBY, J. P. Microeconomia da firma: teoria e prática: 6. ed. Rio de Janeiro: Prentice-Hall do Brasil, 1998.

WALSH, C. E.; STIGLITZ, J. Introdução a microeconomia. 3. ed. Rio de Janeiro: Campus, 2003. WESSELS, W. J. Microeconomia: teoria e aplicações. São Paulo: Saraiva, 2002.

\section{Sites da operadoras}

$<$ www.brasiltelecom.com.br >

$<$ www.claro.com.br>

$<$ www.ctbctelecom.net.br>

$<$ www.sercomtelcelular.com.br $>$
$<$ www.telemar.com.br $>$ $<$ www.telemigcelular.com.br $>$ <www.tim.com.br> $<$ www.vivo.com.br> 